\title{
Analysis and synthesis of factors determining the sintering speed of sinter charge
}

\author{
Serhii Saveliev*, and Mariia Kondratenko \\ Kryvyi Rih National University, Ferrous metals metallurgy \& foundry Department, Kryvyi Rih, Ukraine
}

\begin{abstract}
The main objectives of the study are the analysis and generalization of the parameters that determine the intensity of the sintering, the development of a model of motion of the combustion zone of the sinter charge. As a result of the analysis of the known indicators of the intensity of the sintering process, it was shown that the most representative characteristic of the intensity of the process is the vertical sintering speed, which is an integral part of the complex intensity indicator - the specific productivity of the sintering machine. Many various models of the sintering process have been developed. The author develops a new mathematical model of the motion of the solid fuel combustion zone during sintering of the sinter charge (vertical sintering speed). The model is presented in the form of an expanding network of separate interrelated dependencies (equations), selected as independent submodels. The results of the study using the developed model of the dependence of the vertical sintering speed on the solid fuel content and on the flux content in the charge, are presented. It is concluded that the presented model can be considered as an element of a universal model software complex of the sintering process.
\end{abstract}

\section{Introduction}

At the present stage of development of ferrous metallurgy, sinter is and will remain for a long time the main type of prepared iron ore for the production of pig iron and pig iron, in turn, is the main source material for steel production. Therefore, issues related to improving the efficiency of sinter production are of undoubted practical and scientific interest, are one of the conditions for ensuring a sustainable future for the steel industry. One of the urgent problems of scientific and technological progress in sintering production is to increase its intensity, which means increasing the intensity of the technological process, increasing its speed.

Despite the presence of a rather large number of monographs, articles, scientific and technical conferences devoted to the intensification of the sintering process [13], methods for assessing and indicators of the intensity of the sintering process are not well understood. To date, a detailed analysis of the used indicators of the intensity of the sintering has not been performed, the relationships between them have not been studied, as is done, for example, with respect to the indicators of the intensity of the blast furnace. This situation negatively affects the development of sintering production, since it does not allow a reliable comparison of the intensity of the sintering machines, the sintering rate of sinter charge as well as the development effectiveness of various methods of intensifying the sintering process of the iron ore charge, and the assessment of the reserves for improving the technical and economic indicators of the operation of sinter plants. Since the determining technological operation in the production of sinter is the sintering of the sinter charge, it is precisely on it, in the first place, attention should be paid to solving this problem. This article is devoted to the analytical study and generalization of indicators of the intensity of the sinter process, factors affecting the of a new mathematical model, the movement of the combustion zone of solid fuel of the sinter mixture and its application to assess the influence of various factors on the sintering.

\section{Literature review}

The analysis of indicators of the intensity of the sintering process should begin with the term "intensity of the sintering process" proposed by H. Wendeborne in 1934. It is defined as the ratio of the total heat consumption for heating a material to the amount of heat released during the combustion of carbon in $\mathrm{CO}$ and $\mathrm{CO}_{2}$ and during exothermic reactions, minus the heat for oxide dissociation and evaporation water, the value of which is close to $1.9-2.1$, is essentially a thermal characteristic of the sintering process, depending on the composition of the charge. This indicator is not related to the speed of the sintering process and is not used to assess the intensity of the sintering process.

As a rule $[4$, p. 50; 5, p. 345], the sinter process intensity (sinter machine operation intensity) is estimated using two indicators: 1) specific sinter productivity, measured in tons of suitable sinter, produced from $1 \mathrm{~m}^{2}$ of sintering area of the sinter machine in the "hot" hour (that is, without taking into account the downtime of the sinter

\footnotetext{
* Corresponding author: comher@,bk.ru
} 
machine); 2) the vertical sintering speed, measured by the length of the path traveled by the combustion zone of solid fuel per unit time.

A feature of the sintering process, which must be taken into account when assessing its intensity, is the complex nature of the process, which includes three successive technological stages: 1) preparation of the charge for sintering; 2) sintering of the charge; 3 ) crushing sinter and separation of the return. Each of the stages has both a direct effect on the intensity of the sintering process, and indirectly - through the impact on the subsequent technological stages of sinter production. Based on these considerations, the specific productivity of the sinter machine is a comprehensive indicator of the overall intensity of the sintering process, since all the above stages of the technology of sintering production find expression in it. It is also obvious that the vertical sintering speed directly characterizes only the second stage of the sintering technology and should be considered as one of the components of the overall intensity of the sintering process. The two other components that determine the overall sintering intensity should be considered the bulk mass of the charge and the yield of suitable sinter from the charge, which directly characterize the first and third stages of sintering preparation of the charge for sintering, crushing of sinter and separation from it the return respectively.

We derive a formula relating the general indicator of the intensity of the agglomeration and the private (its components) indicators of intensity. To do this, we will use the following notation: $q_{s}-$ specific productivity of the sinter process according to suitable sinter, $\mathrm{kg} /\left(\mathrm{m}^{2} \mathrm{~s}\right) ; \rho$ - bulk density of the charge, $\mathrm{kg} / \mathrm{m}^{3} ; v$ - vertical sintering speed, $\mathrm{m} / \mathrm{s} ; k$ - yield of suitable sinter from the charge, a fraction of units; then the general indicator of intensity will be a product of its three private components:

$$
q_{s}=\rho \cdot v \cdot k .
$$

Comparison of the three components of the total (complex) indicator of the intensity sintering shows that the most significant and important of them is the vertical sintering speed. The predominant role of the vertical sintering rate in the overall intensity of the sinter process is due to at least three circumstances. Firstly, it is the only component of the general intensity, which has a unit of time in its dimension, without which the concept of intensity loses its physical meaning. Secondly, the practical possibility of sintering intensification is associated, first of all, with an increase in the vertical sintering speed, which in real conditions of sintering production varies widely. The increase in the intensity of the sinter process due to two other components is in most cases limited by the relatively insignificant effect of the composition of the initial charge materials on the bulk density of the charge, as well as the requirements for the quality of the finished sinter. Thirdly, the vertical sintering speed characterizes the process of burning solid fuel of sinter charge, which is the cause of the formation of the melt, which actually ensures the sintering of the charge during the sintering process.
Given the importance of the indicator of the vertical sintering speed of the sinter charge, the practical and theoretical interest is the problem of its calculation definition, for the solution of which today there is no reliable generally accepted technique.

With sufficient accuracy for engineering calculations proposed [6, p. 496] assume that

$$
v_{s}=w_{o} /\left(V_{g} \cdot \rho\right),
$$

where $v_{c}$ - vertical sintering speed, $\mathrm{m} / \mathrm{s}$;

$w_{o}$ - gas filtration rate in the layer, $\mathrm{m} / \mathrm{s}$;

$V_{g}$ - specific yield of sinter gas, $\mathrm{m}^{3} / \mathrm{kg}$ dry charge;

$\rho$ - bulk mass of the sinter charge, $\mathrm{kg} / \mathrm{m}^{3}$.

At the same time, it is known [6, p. 507] that the vertical sintering speed is directly proportional to the gas permeability of the sintered layer $R, \mathrm{~m}^{3} / \mathrm{m}^{2} \mathrm{~min}$, the heat capacity of gases $c_{g}$ and inversely proportional to the apparent heat capacity of the charge $c_{a p . c h}$ :

$$
v_{s}=k\left(c_{g} / c_{\text {ap.ch. }}\right) R,
$$

where $k$ - coefficient of proportionality.

Comparative calculations have established that formula (2) has the greatest versatility and suitability for practical use. Formula (3) is less preferable for the calculated determination of the vertical sintering speed due to the difficulty of accurately taking into account all the factors affecting the apparent heat capacity of the charge and the ambiguity of its determination methods. Moreover, comparing formulas (2) and (3), it should be borne in mind that in the region of a high carbon content in the charge (more than 4\%), despite the decrease in the apparent heat capacity of the charge due to an increase in the mass fraction of carbon in it, the sintering rate decreases [5, p. 170], although according to formula (3) it should increase.

Another indicator characterizing the intensity of sintering of the charge may be the residence time of the charge on the sinter machine (more precisely, over the sintering area of the sinter machine). If we denote the height of the sintering layer of the mixture through $H(\mathrm{~m})$, then in the normal course of the sinter machine, the residence time of the charge on it is determined by the formula

$$
\tau=H / v .
$$

Substituting into the formula (1) instead of the vertical sintering speed its value from formula (4), we obtain a slightly different, more convenient for practical use form of the formula for calculating the specific productivity

$$
q_{s}=\rho \cdot H \cdot k / \tau \text {. }
$$

The main method of theoretical generalization of factors influencing any process is mathematical modeling, which is widely used in various fields, including in the process of agglomeration. Modeling of sintering and firing processes should contain a combination of the most important properties inherent in the objects of study. When constructing models of sintering and firing, it is necessary to take into account the numerous parameters of this process [7]. Important input parameters are: the 
content of the main components in the charge; charge moisture; gas permeability of the charge; charge layer height; air consumption for the process; ignition conditions - hearth temperature (temperature in the space of furnace), ignition temperature (temperature above the surface of the sintered charge), generalized heat treatment temperature. Important output parameters are: sintering speed; composition and temperature of exhaust gases; mass fraction of iron oxide in the finished product; discharge; sintering time. Disturbing effects: change in the composition of the charge; change in the moisture content of the charge; change in the degree of compaction of the charge; change in the height of the charge layer; change in the speed of movement of the sinter; suction of cold air; change in discharge above the ignited layer; change in the fuel-air ratio.

Currently, computer simulation has become widespread, making it possible to process large amounts of information as part of control systems for complex metallurgical processes, which is sinter production. It is believed [8] that the combination of a mathematical model and artificial intelligence is a suitable method for controlling the sintering process.

Deficiencies and limitations of existing mathematical models for determining the performance of sintering machines were identified [9]. These disadvantages depend on a number of technological factors, often interrelated: the speed of movement of the trolleys of the sintering machine, the vertical speed of sintering of the charge, the speed of filtration of air in the layer of the sinter charge. Based on the identified shortcomings and limitations of the existing mathematical models for calculating the performance of sintering machines, the following basic set of technological factors was proposed to develop a mathematical description of the dependence of the performance of the sintering machine on the parameters of the sintering process: general gas-dynamic resistance of the charge layer; the height of the charge layer on the sinter machine; bulk density of the sinter charge; particle size distribution of the sinter charge (the size of the sintered material expressed in terms of the equivalent diameter of the pelletized charge); the duration of the sintering of the charge; sinter charge temperature; sinter charge moisture; return share/yield; carbon content in the sinter charge; the content of fine-grained concentrates in the sinter charge.

A study of the performance indicators of the agglomeration process [10] showed that in laboratory conditions the most objective is a comparative assessment of the productivity of the sinter process by raw charge and yield of sinter with a closed balanced cycle of the return of the constant size. Under production conditions, it is advisable to conduct a comparative assessment of the specific productivity of the sinter process taking into account the total yield of the return or the strength of the sinter.

Based on the characteristics of the sintering process, a long and short term control strategy of sintering burn through point was put forward [11], with the burn through point optimized with a fuzzy controller. Long term control was realized by adjusting bed height or density according to the state of preignition permeability and vertical sintering speed. The rising position of gas temperature was stabilized by adjustment of pallet speed, enabling short term control to be carried out. By using vertical sintering speed to represent bed permeability a prediction model of vertical sintering speed was established. The predicted results are in accordance with calculated values and accuracy of the model is $>95 \%$. The application of system shows that the accuracy of guidance is $>95 \%$ and this system can be used to industry production.

Using the dimensional analysis method, formulas (models) for the efficiency of the sintering process (specific productivity of the sintering machine) were developed from: 1) the gas permeability of the charge; 2 ) the size of the charge; 3 ) a specific load complex equal to the product of the height of the charge layer by the bulk density of the charge; 4) vertical sintering speed [12]. These formulas allow us to calculate the optimal process parameters for specific charge conditions of the sintering process.

The above analysis shows that the intensity of the sintering process (vertical sintering speed) is determined by a combination of factors forming the material composition and structural and gas-dynamic characteristics of the sintered charge layer. Based on this approach, we will carry out mathematical modeling of the process of moving the combustion zone of solid fuel in the sintered layer, taking as a basis formula (2), as the most versatile and suitable for practical use.

\section{Problem formulation}

Based on the foregoing material, the following tasks were set in the work. To develop a new model of the movement of the combustion zone of solid fuel of the sinter charge, characterizing the intensity of the sintering process, and also discuss the possibilities of its application to assess the influence of various factors on the sintering intensity.

\section{Methods}

The following methods were used to solve the tasks: - scientific analysis and generalization of previously performed studies of agglomeration production aimed at determining the factors that determine the sintering rate of the sintering charge;

- theoretical studies of the influence of individual factors that affect the sintering rate of the sintering charge;

- experimental studies of technological techniques what determines the speed of the combustion zone of solid fuel; - mathematical modeling of the process of moving the combustion zone in the sintering layer.

\section{Analysis}

The development of a model for moving the fuel combustion zone in the charge began with the formalization of this process. At the same time, a number of interrelated physical and chemical processes and gasdynamic phenomena were taken into account when preparing the charge for sintering and directly during 
sintering of the sintering charge layer, namely: the formation of the bulk mass of the pelletized charge; gas movement in the sinter layer; burning carbon solid fuel; dissociation of carbonates; the change in gas volume along the height of the sintered layer as a result of heat and mass transfer processes.

The main structural elements of the model are charge parameters and gas parameters that characterize the initial state of the system.

The parameters of the sinter charge, characterizing the initial state of the system, include: the content of the individual components of the charge; mass fraction of carbon in fuel, $\mathrm{CaO}$ in limestone and $\mathrm{CaO}_{\text {act }}$ in lime; fractional composition of fuel and charge; charge layer height.

The input gas parameters established during the calculations for a specific case of sintering are: the composition of the gas at the entrance to the layer; air suction rate through the sinter layer; specific air consumption for sintering.

The processes under consideration are grouped into the following subsystems: gas filtration rates in the layer; specific output of sinter gas; bulk density of the pelletized charge.

The output parameter is the vertical sintering speed of the sinter charge.

Thus, the structural diagram of a mathematical model can be represented in the form of Figure 1.

Having designated the main structural elements of the model, we will carry out the development and idealization of its mathematical description.

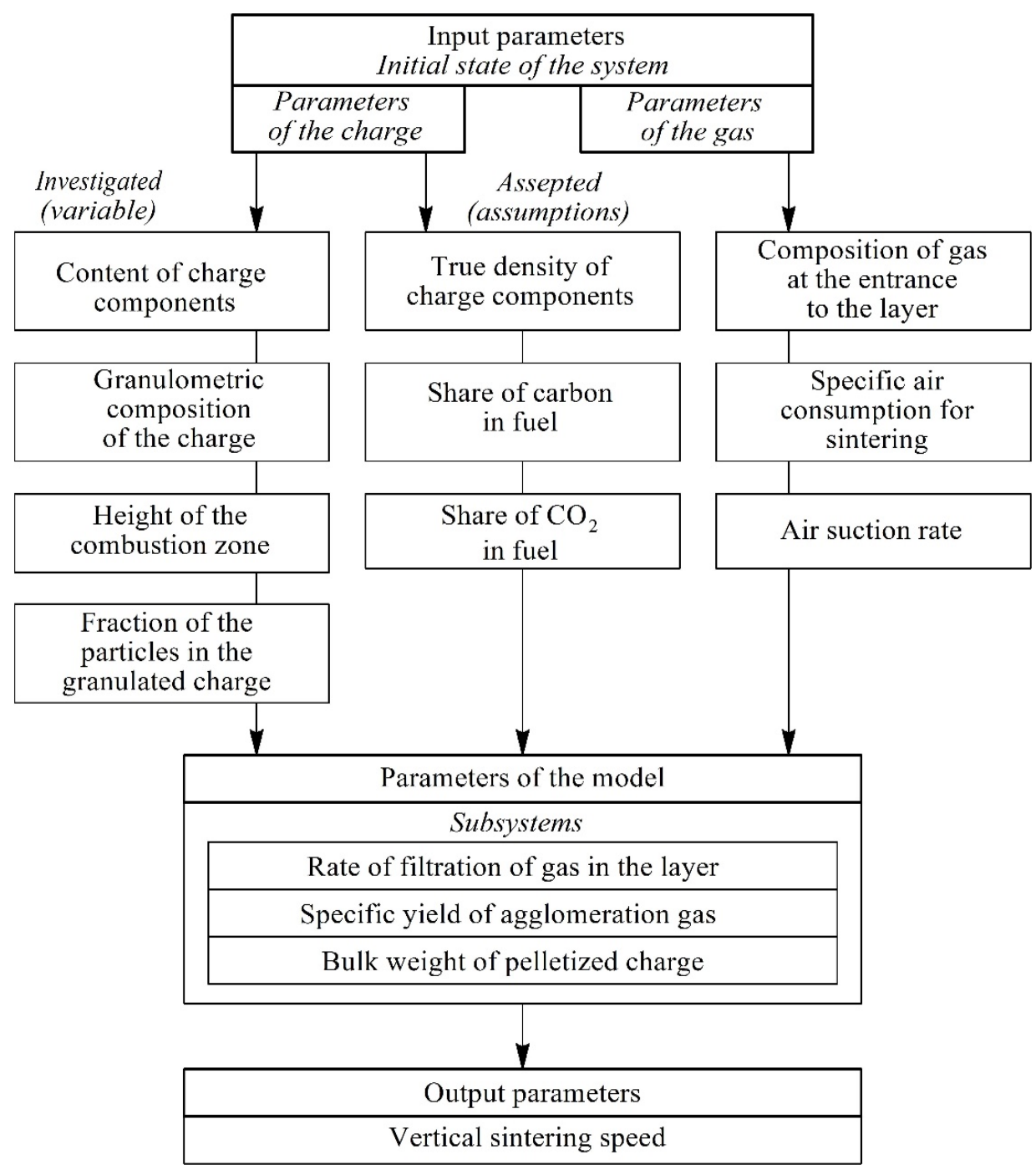

Fig. 1. Structural scheme of mathematical model of movement of the combustion zone solid fuel in the sintering layer of the charge (vertical sintering speed).

In developing the mathematical description of the conceptual model of the vertical sintering speed, the following basic assumptions were made:

1) the charge is presented in the form of ore, fuel, flux particles and the return of spherical shape;
2) carbon burning occurs according to the reactions $\mathrm{C}+0,5 \mathrm{O}_{2}=\mathrm{CO}, \mathrm{C}+\mathrm{O}_{2}=\mathrm{CO}_{2}$;

3 ) the granulometric composition of the pelletized charge is determined by the mass fraction of individual fractions of fineness in the initial charge and the content of binder additives under optimal pelletizing conditions; 
4) the gas flow is evenly distributed over the cross section of the layer, and its flow rate over time is unchanged;

5) heat loss to the environment is negligible;

6) the rate of combustion of a fuel particle is determined by its size and true density;

7) the fuel burns in a layer of inert materials and its particles are equidistant from each other.

When developing submodels for determining the gas filtration rate, the following assumptions were made: - the specific air consumption for sintering the non-fluxed charge from hematite ores and magnetite concentrates is $0.35-0.40 \mathrm{~m}^{3} / \mathrm{kg}$ (in the practice of sintering of iron ore materials in most cases the carbon content does not exceed $5 \%$ and the specific air consumption remains almost the same [5, p. 111]). This assumption was made when calculating the specific yield of sinter gas (based on typical air flow);

- the height of the combustion zone for typical sintering conditions (fuel with a size of $0-3 \mathrm{~mm}$ ) is $1 / 10$ of the total height of the sintered layer $[5$, p. 65, 68];

- the rate of gas leakage through the sinter layer is $0.35 \mathrm{~m} / \mathrm{s}$. These assumptions were made when calculating the dependence of the burning rate of fuel particles on various factors.

The sinter gas specific output subsystem takes into account the flow of carbon into the charge with fuel and limestone, as well as the characteristics of the gas entering the layer.

When developing the formula for the mathematical description of the specific output of the sintering gas, the following assumption was made: the parameters of the air supplied to the layer correspond to atmospheric at a temperature of $25^{\circ} \mathrm{C}$, which is typical for typical sintering conditions.

The bulk density subsystem of the pelletized charge takes into account the characteristics of the charge (chemical and particle size distribution), as well as the fines content in the charge and the ratio of the diameters of small and large particles.

When developing a submodel of the bulk density of the charge, the following assumptions were made:

- the density of the components of the charge is unchanged;

- during pelletizing, the number of relatively large granules increases as a result of rolling a small fraction onto the embryos.

The mathematical model of the movement of the combustion zone in the sintered layer is presented in the form of an expanding network of separate interrelated dependencies (equations), identified as independent submodels.

We set the range of input data for typical sintering conditions:

- the height of the sintered layer does not exceed $500 \mathrm{~mm}$;

- the size of solid fuel (coke breeze) does not exceed $5 \mathrm{~mm}$.

- the chemical composition of the mixture has a number of limitations on the input data on the content of limestone and solid fuel. The limestone content should be in the range from 0 to $25 \%$, since the nature of the curve of the temperature change of the air entering the combustion zone with a high content of fluxing additives has not been fully studied. The content of solid fuel (coke breeze) should not exceed $8 \%$, since some mathematical dependencies expressed in the corresponding submodels will be unfair;

- the density of the components of the charge and air parameters are taken according to the reference data. The density of the charge components $\left(\mathrm{kg} / \mathrm{m}^{3}\right)$ : magnetite concentrate 4.7 ; hematite ore 4.5 ; coke breeze 1.6 ; limestone 1.0 ; dolomitic limestone 1.1 ; lime 2.0; refund 3.5; manganese ore 4.2 .

The adequacy check and adaptation of the model for determining the vertical rate of sintering of the charge was carried out by comparing the simulation results with experimental data obtained in laboratory and industrial studies conducted at the sinter plant of MPP of PJSC "ArcelorMittal Kryvyi Rih" - mining and processing plant of public joint stock company "ArcelorMittal Kryvyi Rih".

The research was carried out in the laboratory. The ore part of the charge consisted of MPP concentrate (90\%) and Kryvyi Rih agglomeration ore $(10 \%)$. For fluxing, a mixture of ordinary and dolomite limestone in a ratio of $3: 2$ and quicklime obtained by firing this mixture of limestones was used. The degree of calcination of limestone was $70 \%$, the size- $3-0 \mathrm{~mm}$. As a solid fuel, a mixture of coke and anthracite block in a ratio of $1: 1$ was used. The content of fuel, return and additional manganese ore in the charge was 4,18 and $2 \%$, respectively. The height of the sintered layer was $0.28 \mathrm{~m}$, the charge basicity was 1.3.

The calculation of the numerical methods of the model was carried out using the Microsoft Excel software application. According to the experimental data, the vertical sintering speed for a charge with such parameters is $26 \mathrm{~mm} / \mathrm{min}$., and according to the results of calculations using the model it is $26.65 \mathrm{~mm} / \mathrm{min}$., which indicates the coincidence of the model properties with the corresponding properties of the simulated object.

The adequacy of individual submodels of the dependence of certain process parameters on various factors was also verified by comparing the calculated and experimental values. So, determining the height of the combustion zone using the appropriate submodel gives adequate characteristic dependencies, shown in Figure 2.

Thus, verification of the adequacy of the model showed the coincidence of the properties of the model and the corresponding properties of the simulated object.

\section{Discussion}

In order to discuss the simulation results, a study was made of factors affecting the agglomeration rate using the developed model. Using the created model, we studied the dependence of the vertical sintering speed on the solid fuel content at various heights of the sintered layer. The initial parameters of the charge: the basicity of the charge of 1.0; air suction rate $0.35 \mathrm{~m} / \mathrm{s}$. Adjustable parameters: solid fuel content in the charge 1-8 \%; charge layer height 200-500 $\mathrm{mm}$. 


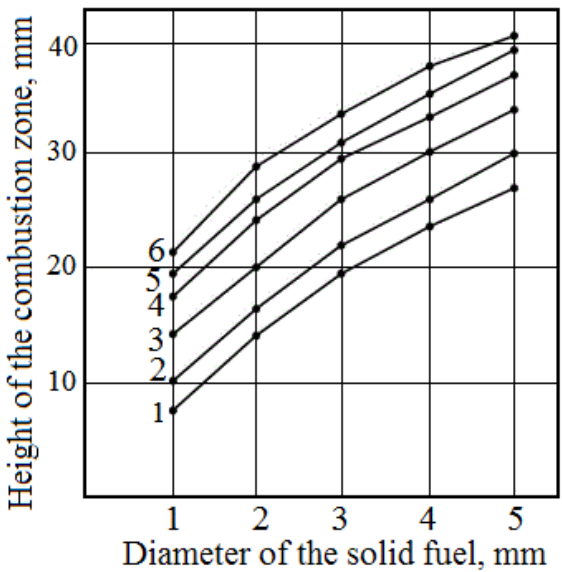

Fig. 2. The dependence the height of the combustion zone from the diameter of the solid fuel at different layer heights the height of the sintered layer, mm: $1-150,2-200,3-300$, $4-400,5-500,6-600$

The research results (Fig. 3) show an extreme dependence of the vertical sintering speed on the fuel content (the maximum is in the region of $3.5-3.7 \% \mathrm{C}$ ), as well as an increase in the vertical sintering speed with a decrease in the layer height, which corresponds to the known laws of the theory of the sintering process.

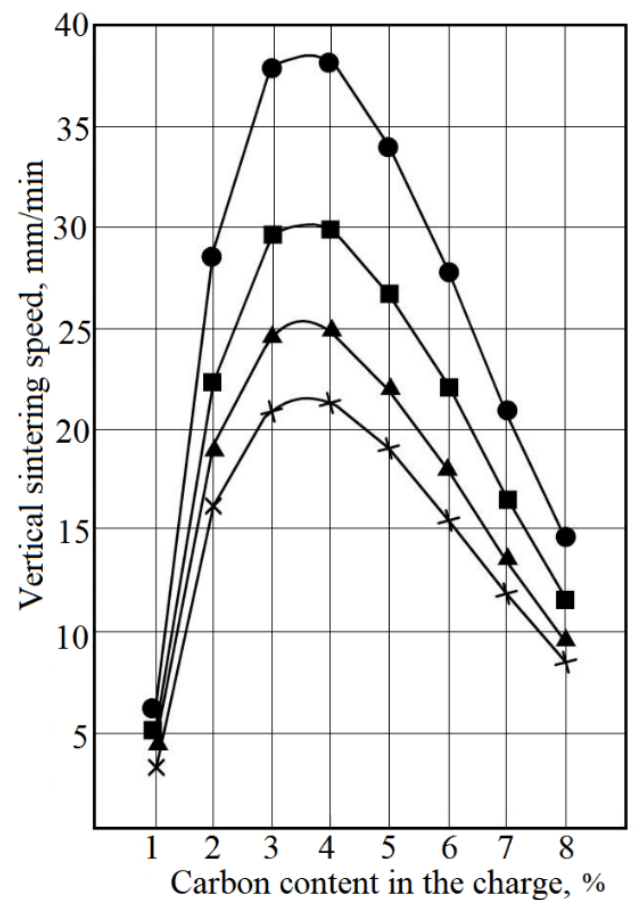

Fig. 3. The dependence of the vertical sintering speed on the fuel content in the charge at different layer heights, $\mathrm{m}: \bullet-0,2$; - $-0,3 ; \boldsymbol{\Delta}-0,4 ; \times-0,5$.

A model study of the dependence of the vertical sintering rate on the flux content (a mixture of limestone and dolomitic limestone) at different heights of the sintering layer was carried out taking a constant solid fuel content of $4.0 \%$. Adjustable parameters: charge basicity 0-2.5; charge layer height 200-500 $\mathrm{mm}$.

The research results (Fig. 4) show that limestone is an intensifier of the agglomeration process. With an increase in the basicity of the charge, the sintering rate increases by $12-14 \%$ (a larger value corresponds to the lowest layer height).

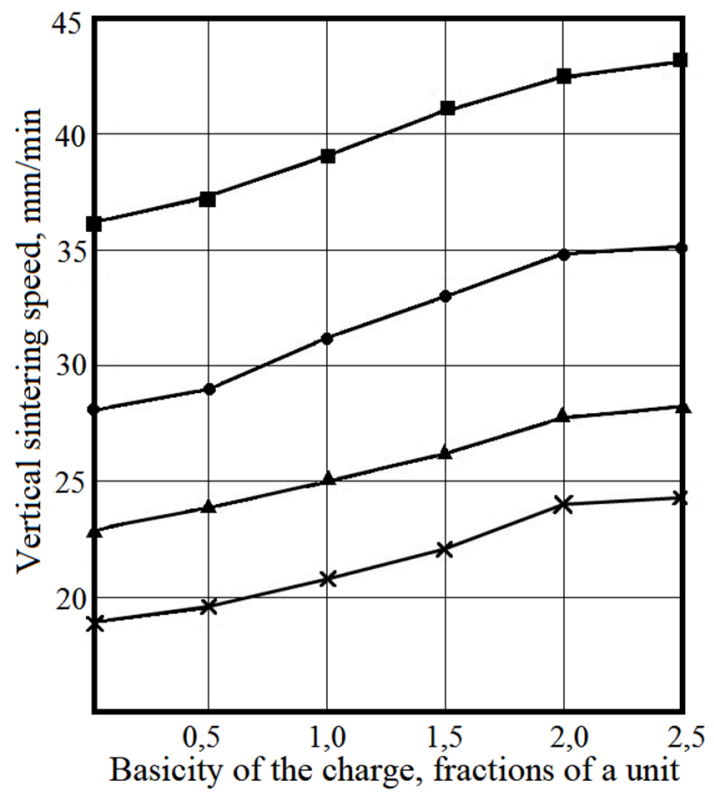

Fig. 4. The dependence of the vertical sintering speed on the basicity of the charge at different layer heights, $m:-0,2$; $\bullet-0,3 ; \boldsymbol{\Delta}-0,4 ; \times-0,5$.

The negative effect of limestone supply is to reduce the porosity of the layer of pelletized charge due to an increase in the fraction of the fine fraction in it. The quantitative nature of this dependence is also presented in the corresponding submodel. With an increase in the limestone content in the charge to $25 \%$, the fraction of fines in the dry charge increases to $85 \%$. However, this improves the gas permeability of the charge directly during the sintering process. One of the reasons for this phenomenon is obviously a decrease in the content of relatively large granules (more than 10-12 mm) in the pelletized charge, which are prone to destruction in the drying and waterlogging zones during the sintering process.

A positive effect on the gas permeability of the sintered layer with increasing basicity also has a decrease in the optimal moisture content of the charge, since this increases the specific consumption of the heat carrier gas for moisture evaporation, which helps to preserve the strength of the granules.

Another explanation for the increase in the vertical sintering rate is possible, based on calculations of the apparent heat capacity of the charge at different limestone contents. Taking into account the heat that is released during the formation of calcium ferrites, an increase in the proportion of limestone in the charge reduces the maximum temperature in the solid fuel combustion zone, which is accompanied by an increase in gas filtration through the layer.

\section{Conclusions}

Thus, the performed studies show that the developed mathematical model of the process of moving the combustion zone of solid fuel in the sinter layer of the 
sinter charge allows us to study the dependence of the vertical sintering speed on the parameters of the material composition and structural and gas-dynamic characteristics of the sinter charge layer. The obtained numerical description of the process is a local dynamic model, the adequacy of which is confirmed by the results of theoretical and experimental studies. Despite the local nature of the developed model, due to its versatility, it takes into account the main material and structural parameters of the sintering process, which affect the speed of movement of the solid fuel combustion zone, and as such can be considered as element of the universal software package of sintering process model [7].

The scientific novelty of the work consists in the development of a model that for the first time allows us to determine the speed of the agglomeration process without conducting experimental sintering, as well as to investigate the influence of the composition and structural characteristics of the charge on the sintering intensity.

The obtained results create prerequisites for choosing the sintering technology that provides the highest sintering speed and, consequently, the productivity of the sintering machine for specific sintering conditions. This fact is an important factor in ensuring the economic security of sintering production, a condition for its sustainable future.

\section{References}

1. S.V. Krivenko, A.A. Tomash, V.P. Russkih, Metal and casting of Ukraine 7-8, 63-68 (2009)

2. V.V. Plotnikov, L.N. Saitgareev. Announcer of priazovskiy state technical university. Series: engineering sciences 25, 41-46 (2012)

3. P. Besta, P. Wicher. The optimization of the production of sinter as the feedstock of the blast furnace process. Metallurgy 56 1-2, 131-134 (2017)

4. Sintering plant at a glance (2015), https://vdocuments.mx/sintering-plant-at-aglance.html. Accessed 28 Mar 2020

5. V.I. Korotich, Yu.A. Frolov, G.N. Bezdezhskiy, Agglomeration of ore materials (UGTU-UPI, Ekaterinburg, 2003)

6. M.G. Ladyigichev et al., in Raw material for ferrous metallurgy, ed. by V.M. Chizhikov, vol. 1 (Mashinostroenie-1, Moscow, 2001), p. 896

7. S.G. Saveliev, Ya.A. Stoykova, Mathematical modeling in the study of the production of iron-ore raw material. Mountain announcer 95, 99-104 (2012)

8. F. Xiaohui, C. Xuling, Yi Wang, Expert System for Sintering Process Control, in Expert Systems, ed. by P. Vizureanu (IntechOpen, London, 2010). doi:10.5772/7073

9. D.R. Ganin, V.G. Druzhkov, A.A. Panyichev, A.N. Shapovalov, Review and analysis of mathematical models for calculating the performance of an agglomeration machine. Theory and technology of metallurgical production 2 (15), 20-25 (2014)
10. V.B. Semakova, E.I. Pilyugin, Research of performance indicators of sintering process, Announcer of Priazovskiy state technical university. Series: engineering sciences 30(), 41-50 (2015)

11. X.L. Chen, X.H. Fan, Y. Wang et al., Control guidance system for sintering burn through point. Ironmaking \& Steelmaking: Processes, Products and Applications 36(3), 209-211 (2009). doi:10.1179/174328107X155367

12. A.A. Panychev, A.P. Nikonova, Parameters of sintering of Michael and Swan concentrates. Announcer of Moscow State Technical University the name of G.I. Nosov 4, 18-22 (2009) 